\title{
Effet des amendements organiques sur le développement du riz (Oryza sativa $L$.) dans les bas- fonds sulfato-acides en zone sud-soudanienne au Sénégal.
}

\author{
Yves Paterne SAGNA ${ }^{*}$, Siré DIEDHIOU1, Arfang Ousmane Kémo GOUDIABY1, Yaya DIATTA', \\ Mariama Dalanda DIALLO², Ibrahima NDOYE ${ }^{3}$ \\ 1 Université Assane Seck Ziguinchor ; Département d’Agroforesterie ; Sénégal \\ 2Université Gaston Berger ; Unité de Formation et de Recherche ; Sciences Agronomiques, de l'Aquaculture et de \\ Technologies Alimentaires ; Saint Louis, Sénégal \\ 3Université Cheikh Anta Diop ; Département de Biologie Végétale ; Sénégal \\ ${ }^{*}$ Corresponding author, e-mail: y.sagna89@gmail.com
}

Original submitted on $10^{\text {th }}$ September 2019. Published online at www.m.elewa.org/journals/ on $31^{\text {st }}$ December 2019 https://doi.org/10.35759/JABs.v144.11

\begin{abstract}
RESUME
Objectifs: L'acidification des rizières conduit à une libération excessive de fer entrainant la toxicité ferreuse qui limite le rendement du riz. L'apport d'amendements pourrait améliorer le rendement du riz. L'objectif de cette étude était de déterminer l'effet des amendements organiques sur le développement du riz dans les bas-fonds ferrugineux acides.

Méthodologie et résultats: Un dispositif randomisé a été mis en place dans la zone avec toxicité ferreuse (zone Tox) et dans la zone sans toxicité ferreuse (zone STox) avec trois amendements (biochar, compost, et témoin) et 4 variétés de riz. En zone Tox, après 90 jours, l'amendement au compost a entrainé une baisse du pH : 3,93 pour l'horizon $0-10 \mathrm{~cm}$ et 3,89 pour l'horizon $10-30 \mathrm{~cm}$; cependant, le nombre de talles a augmenté $(p<0.01)$. Dans la zone Tox, l'apport de biochar a augmenté significativement la hauteur moyenne de la variété Suakoko $8(p<0,01)$.

Conclusions et applications des résultats: L'utilisation du compost bien qu'ayant abouti à l'augmentation du nombre de talles, devrait se faire de manière prudente et s'accompagner si possible de matière basique comme de la cendre permettant d'élever le pH des rizières. II serait intéressant de suivre également l'effet de l'apport du biochar dans les rizières à long terme. Ces amendements organiques pourraient à long terme augmenter le $\mathrm{pH}$ du sol des rizières et rendre ainsi les nutriments disponibles pour la plante ; ceci contribuera à de meilleurs rendements du riz.
\end{abstract}

Mots clés: Oryza sativa L., Biochar, Compost, Toxicité ferreuse, Bas-fonds. 
Development of rice (Oryza sativa L.) amended with compost and biochar in the sulfato-acid lowlands in southern Senegal.

\begin{abstract}
Objectives: Paddy rice acidification leads to an excessive release of ferrous iron causing the iron toxicity of rice which limits rice yield. Adding organic amendment could improve rice yield. This study was done to determine the effect of organic amendments on rice development in acid ferruginous lowlands.

Methodology and results: A split-plot design was set up in the area with iron toxicity (Tox zone) and in the area without iron toxicity (Stox zone) with three amendments (biochar, compost, and control) and 4 varieties of rice. In the Tox zone, after 90 days, compost amendment led to a drop in $\mathrm{pH}: 3.93$ for the 0-10 $\mathrm{cm}$ horizon and 3.89 for the $10-30 \mathrm{~cm}$ horizon; however, the number of tillers increased $(p<0.01)$. In the Tox zone, the supply of biochar significantly increased the average height of the Suakoko 8 variety $(p$ $<0.01)$.

Conclusions and application of the results: The use of compost, although having led to an increase in the number of tillers, should be done carefully and be accompanied, if possible, by material such as ash, which raises the $\mathrm{pH}$ in rice fields. It would also be interesting to follow the long-term effect of biochar in rice fields. Amendments of compost and biochar could in the long run increase the $\mathrm{pH}$ of rice fields and thus make the nutrients available to the plant; this will contribute to better rice yields.
\end{abstract}

Keywords: O. sativa L., Biochar, compost, ferrous Toxicity , paddy.

\section{INTRODUCTION}

La riziculture de bas-fond est confrontée à des contraintes abiotiques ne permettant pas une exploitation rationnelle et durable de ses potentialités surtout en Casamance. Une étude menée par l'ADRAO en 2001 a révélé que jusqu'à $60 \%$ des zones de bas-fonds rizicoles en Afrique de l'Ouest sont exposées aux phénomènes de toxicité ferreuse et/ou de salinité. La perte moyenne de rendement due à ces phénomènes atteint dans certaines zones les $100 \%$. Dans le contexte agricole et démographique actuel, il urge d'identifier ou de concevoir des pratiques favorables afin d'améliorer de manière durable la fertilité minérale des sols. L'enfouissement du charbon végétal (biochar) dans les sols est proposé comme une stratégie durable pour à la fois atténuer les changements climatiques et augmenter les productions agricoles (Laird, 2008; Biederman \& Harpole, 2013). En effet, l'amendement modifie les caractéristiques physiques, chimiques et biologiques des sols à long terme vu le temps de résidence et de décomposition de la matière organique dans les sols (Criscuoli, 2016). Selon la littérature, l'amendement au biochar améliorerait les propriétés physiques du sol, telles que la masse volumique apparente, la porosité, la capacité de rétention en eau, la stabilité et la formation d'agrégats (Ding et al., 2016). Grâce à ces amendements, différents auteurs ont observé une augmentation de la production de biomasse ou des rendements (Biederman \& Harpole, 2013). L'amélioration de la fertilité du sol à travers l'amendement au biochar influence la croissance et le développement des plantes car les communautés microbiennes du sol qui interagissent avec les racines sont affectées par la présence de biochar (Dai et al., 2017; Kolton et al., 2017). Laurin-Lanctôt, (2015) ont montré aussi que l'amendement au biochar des sols biologiques a entrainé une augmentation significative des concentrations minérales dans le sol pour certains macroéléments $\left(\mathrm{NO}^{3-}, \mathrm{P}\right)$ et certains oligoéléments $\left(\mathrm{Fe}^{2+}, \mathrm{Cu}^{2+}, \mathrm{Mn}^{2+}\right)$. C'est dans cette optique que la présente étude a été entreprise dans le but de déterminer l'effet du biochar et du compost sur le développement du riz dans les basfonds sulfato-acides du village de Diatock en Basse Casamance. 


\section{MATERIEL ET METHODES}

Présentation de la zone d'étude : L'essai a été réalisé au Sud du Sénégal, dans le village de Diatock, région de Ziguinchor. La zone expérimentale est située en bordure du fleuve Casamance, zone où est essentiellement pratiquée la riziculture de bas-fonds. Le climat est du type Sud-soudano côtier avec une température moyenne annuelle diurne de $35^{\circ} \mathrm{C}$ (PLD, 2010). La pluviométrie moyenne annuelle tourne autour de $1255 \mathrm{~mm}$ et s'étend de Juillet en Septembre.

Matériel végétal et amendements organiques ॥ Le matériel végétal utilisé est constitué de 04 variétés de riz homologuées et résistantes à la toxicité ferreuse (DJ 684 D, Bouaké 189, Suakoko 8 et FAROX 521-356$\mathrm{H1}$ ). Ces variétés ont été fournies par l'Institut Sénégalaise de Recherches Agricole ISRA/CRA de Djibélor, Ziguinchor. Le biochar et les résidus décomposés de pomme d'anacarde (compost anacarde) ont été utilisés. Le compost d'anacarde est obtenu à partir de la pomme d'acajou pressée et décomposée. Quant au biochar, il est issu de la pyrolyse incomplète de matière végétale.

Dispositif expérimental: Le dispositif expérimental est un split plot installé dans deux zones: zone sans toxicité ferreuse et zone avec toxicité ferreuse. Dans chacune de ces zones, un premier niveau aléatoire a été déterminé pour le facteur amendement (biochar, compost et témoin), répété trois fois pour un total de neuf (09) parcelles (figure 1). Un deuxième niveau de disposition aléatoire en factoriel a été appliqué avec le facteur variété de riz avec 4 niveaux (DJ $684 \mathrm{D}$, BOUAKE 189, SUAKOKO 8, FAROX 521-356-H1). Au total, $4^{*} 3^{*} 3=36$ traitements par zone ont été obtenus.

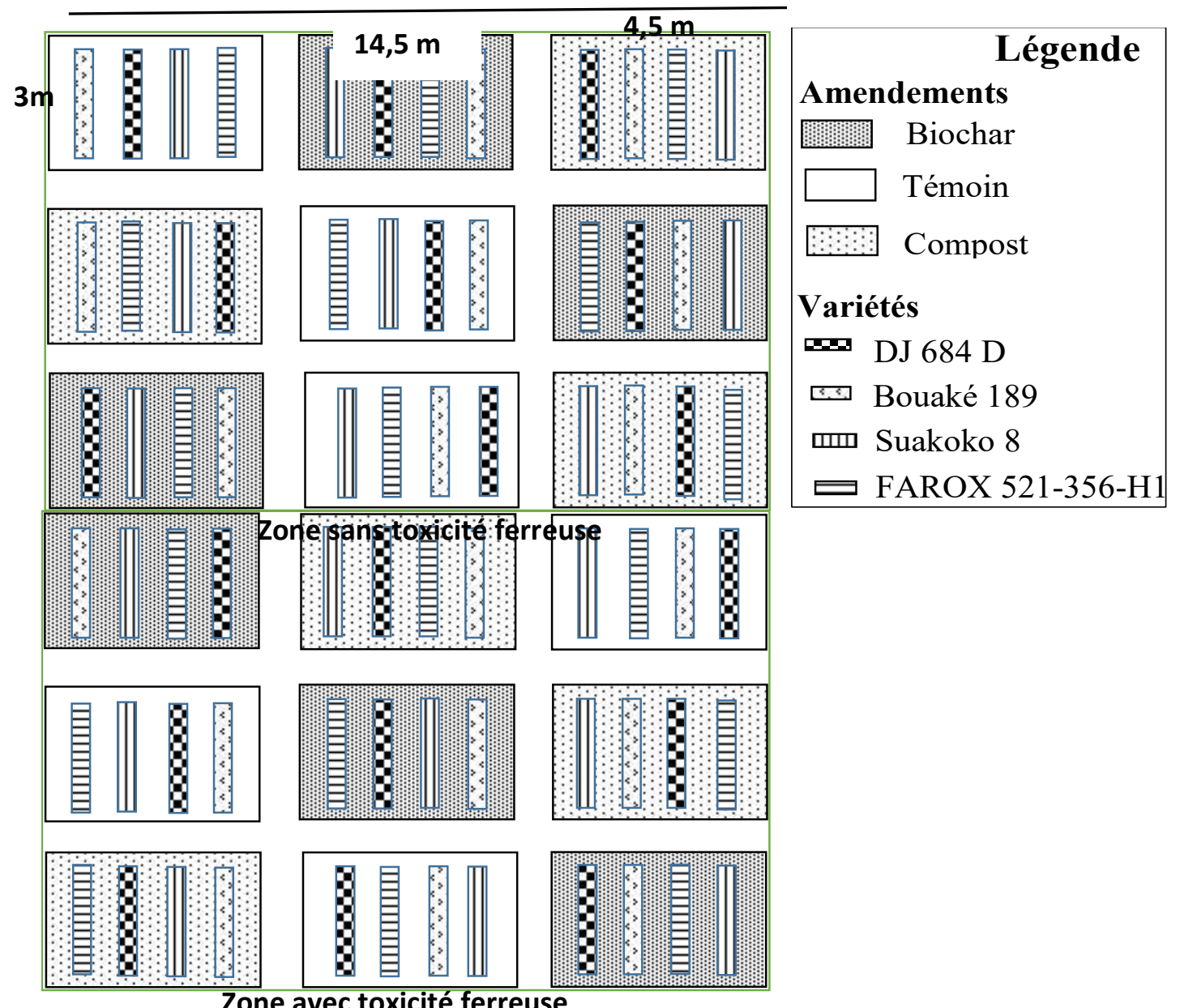

Figure 1 : Dispositif expérimental 
Conduite de l'essai : Le semis à la pépinière a été effectué à la volée sur des billons en plateau à la 3ème semaine de Juillet 2017. Le labour et l'amendement des rizières sont réalisés une semaine avant le repiquage du riz. Des billons de $1 \mathrm{~m}^{2}\left(2^{*} 0,5 \mathrm{~m}\right)$ ont été constitués avec une distance de $0,5 \mathrm{~m}$ entre les billons. Le compost d'anacarde et le biochar sont enfouis à raison $10 \mathrm{~kg} \cdot \mathrm{m}^{-2}$ au moment du labour. Trois lignes de semis distantes de $0.20 \mathrm{~m}$ dans le sens de la longueur des billons ont été définies sur chaque billon. Après 30 jours de culture en pépinière, les plants de riz sont repiqués sur ces billons de rizière à raison d'un (01) plant par poquet. L'écartement entre les poquets sur une même ligne et entre les lignes sur le même billon est de $20 \mathrm{~cm}$. Les plants de riz ont été repiqués sur 11 poquets par ligne pour un total de 33 plants par billon. Un désherbage manuel a été effectué à 15 jours après repiquage (JAR) et à $30 \mathrm{JAR}$.

Collecte des données : La collecte des échantillons de sol a été effectuée à deux niveaux de profondeur : $0-10 \mathrm{~cm}$ et $10-30 \mathrm{~cm}$. Les échantillons sont ensuite séchés à l'air libre pendant 72 heures. De l'eau distillée est ensuite ajoutée aux sous-échantillons de $10 \mathrm{~g}$ de sol pour la mesure du $\mathrm{pH}$. Le taux de survie du riz a été déterminé par comptage du nombre total de plants

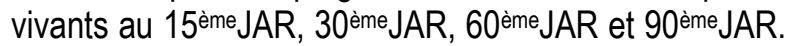
La collecte des données des autres paramètres a été effectuée en choisissant 15 plants par billon et par variété (05 plants par ligne). Un comptage du nombre de talles a été effectué à 60 JAR et la moyenne a en été déduite par la suite. Pour le diamètre moyen, la mesure a été effectuée à 60 JAR correspondant au tallage maximum des plants. Le diamètre a été mesuré au niveau du collet des plants à l'aide d'un pied à coulisse. A 90 jours après repiquage, la hauteur des plants a été mesurée et la biomasse a été collectée. La biomasse a été séchée à l'étuve à $70^{\circ} \mathrm{C}$ pendant 72 heures avant pesage. Le poids moyen des panicules ainsi que le poids des milles grains ont été déterminés. Le rendement a été calculé en utilisant la formule de Lacharme, (2001)

Rendement $=\mathrm{NP} / \mathrm{ha}{ }^{*} \mathrm{NT} / \mathrm{P}^{*} \mathrm{Npa} / \mathrm{T}^{*} \mathrm{NG} / \mathrm{Pa}{ }^{*} \mathrm{PG}$

Avec NP/ha $=$ nombre de plants/hectare $=\mathrm{NP} / \mathrm{m}^{2 \star} 10$ 000

$\mathrm{NT} / \mathrm{P}=$ nombre de talles/pieds $; \mathrm{NPa} / \mathrm{T}=$ nombre de panicule/talle ;

$\mathrm{NG} / \mathrm{Pa}=$ nombre de grains/panicules $; \mathrm{PG}=$ poids d'un grain.

Traitement et analyse des données: Les données collectées ont été soumises à une analyse de variance (ANOVA) à l'aide du logiciel XLSTAT 2014 version 5.03. Les moyennes de chaque variété suivant les paramètres étudiés sont par la suite soumises à une Analyse en Composante Principale (ACP). Le test de Fisher a été utilisé pour la comparaison des moyennes au seuil de probabilité de $5 \%$.

l'amendement au compost au niveau des horizons 0 $10 \mathrm{~cm}$ et $10-30 \mathrm{~cm}$ dans les deux zones. Au niveau de la zone STox, le pH des sols non-amendés (témoin) a significativement baissé aussi par rapport à l'amendement au biochar.

\section{RESULTATS ET DISCUSSION}

Effet des amendements organiques sur le $\mathrm{pH}$ du sol : II n'y a pas eu de variation significative du pH ( $p$ > 0.05 ) quel que soit l'amendement ou la variété dans les 60 jours après repiquage. Cependant, le $\mathrm{pH}$ a significativement diminué à 90 JAR avec

Tableau 1: Variation du pH des sols prélevés à différentes profondeurs en fonction des amendements et du temps dans les zones STox et Tox.

\begin{tabular}{|c|c|c|c|}
\hline \multicolumn{4}{|c|}{ Zone sans toxicité ferreuse } \\
\hline Dates & Amendements & Horizon $0-10 \mathrm{~cm}$ & Horizon $10-30 \mathrm{~cm}$ \\
\hline \multirow{3}{*}{ O JAR } & Biochar & $5,64 a^{*}$ & 5,05 a \\
\hline & Compost & $5,57 \mathbf{a}$ & 5,34 a \\
\hline & Témoin & 5,57 a & $5,21 \mathrm{a}$ \\
\hline \multirow{3}{*}{15 JAR } & Biochar & 5,70 a & 5,58 a \\
\hline & Compost & $5,77 \mathbf{a}$ & 5,65 a \\
\hline & Témoin & $5,75 a$ & 5,67 a \\
\hline \multirow{2}{*}{$60 \mathrm{JAR}$} & Biochar & $4,80 \mathrm{a}$ & 4,75 a \\
\hline & Compost & $4,53 \mathbf{a}$ & 4,59 a \\
\hline
\end{tabular}



dans les bas-fonds sulfato-acides en zone sud-soudanienne au Sénégal.

\begin{tabular}{|c|c|c|c|}
\hline & Témoin & 4,70 a & 4,70 a \\
\hline \multirow{3}{*}{$90 \mathrm{JAR}$} & Biochar & 4,64 a & 4,38 a \\
\hline & Compost & $3,93 \mathbf{b}$ & $3,89 \mathbf{b}$ \\
\hline & Témoin & $3,73 \mathbf{b}$ & $4,44 \mathbf{a}$ \\
\hline \multicolumn{4}{|c|}{ Zone avec toxicité ferreuse } \\
\hline \multirow{3}{*}{ O JAR } & Biochar & 4,84 a & 4,41 a \\
\hline & Compost & 5,20 a & 5,11 a \\
\hline & Témoin & 4,86 a & 4,70 a \\
\hline \multirow{3}{*}{$15 \mathrm{JAR}$} & Biochar & 5,66 a & 5,90 a \\
\hline & Compost & 5,73 a & 5,79 a \\
\hline & Témoin & 5,66 a & 5,69 a \\
\hline \multirow{3}{*}{$60 \mathrm{JAR}$} & Biochar & 4,33 a & 4,47 a \\
\hline & Compost & 4,30 a & 4,25 a \\
\hline & Témoin & 4,11 a & 4,15 a \\
\hline \multirow{3}{*}{$90 \mathrm{JAR}$} & Biochar & 3,47 a & 3,85 a \\
\hline & Compost & 3,89 a & 3,58 a \\
\hline & Témoin & 4,06 a & 3,77 a \\
\hline
\end{tabular}

*Les valeurs de la même colonne pour une même date présentant les mêmes lettres ne sont pas statistiquement différentes au seuil LSD de $5 \%$ selon le test de Fisher.

Effet des amendements organiques sur le taux de survie des plants: II n'y a pas de différence significative pour le taux de survie ni entre les amendements $(P>0,05)$, ni entre les variétés $(P>$ $0,05)$ dans les deux zones jusqu'à 30 JAR. Ce n'est qu'à 60 JAR et à 90 JAR pour l'amendement au compost et pour la variété DJ $684 \mathrm{D}$ que l'on note une mortalité significativement plus élevée en zone Tox $(69.2 \%)$ comparée à la zone STox (98.9\%) (Tableau 2).

Tableau 2: Taux de survie des plants à différentes étapes de quatre variétés de riz cultivées en zone STox et Tox en fonction des amendements

\begin{tabular}{lllll}
\hline \multicolumn{4}{l}{ Zone sans toxicité ferreuse } & \\
\hline Variétés & Etapes & Témoin & Biochar & Compost \\
\hline \multirow{3}{*}{ DJ 684 D } & 15 JAR & $100 \mathrm{a}^{*}$ & $100 \mathrm{a}$ & $100 \mathrm{a}$ \\
& 30 JAR & $96,9 \mathrm{a}$ & $98,9 \mathrm{a}$ & $100 \mathrm{a}$ \\
& 60 JAR & $96,9 \mathrm{a}$ & $98,9 \mathrm{a}$ & $98,9 \mathrm{a}$ \\
& 90 JAR & $96,9 \mathrm{a}$ & $98,9 \mathrm{a}$ & $98,9 \mathrm{a}$ \\
\hline \multirow{5}{*}{ Suakoko 8 } & 15 JAR & $100 \mathrm{a}$ & $98,9 \mathrm{a}$ & $100 \mathrm{a}$ \\
& 30 JAR & $100 \mathrm{a}$ & $98,9 \mathrm{a}$ & $100 \mathrm{a}$ \\
& 60 JAR & $100 \mathrm{a}$ & $98,9 \mathrm{a}$ & $100 \mathrm{a}$ \\
Bouaké 189 & 90 JAR & $100 \mathrm{a}$ & $98,9 \mathrm{a}$ & $100 \mathrm{a}$ \\
& 15 JAR & $100 \mathrm{a}$ & $100 \mathrm{a}$ & $100 \mathrm{a}$ \\
& 30 JAR & $100 \mathrm{a}$ & $100 \mathrm{a}$ & $100 \mathrm{a}$ \\
& 60 JAR & $98,9 \mathrm{a}$ & $100 \mathrm{a}$ & $100 \mathrm{a}$ \\
FAROX 521-356-H1 & 90 JAR & $98,9 \mathrm{a}$ & $100 \mathrm{a}$ & $100 \mathrm{a}$ \\
& 15 JAR & $100 \mathrm{a}$ & $100 \mathrm{a}$ & $100 \mathrm{a}$ \\
& 30 JAR & $100 \mathrm{a}$ & $100 \mathrm{a}$ & $100 \mathrm{a}$ \\
\hline
\end{tabular}




\begin{tabular}{|c|c|c|c|c|}
\hline & 90 JAR & $100 \mathrm{a}$ & $100 a$ & $100 \mathrm{a}$ \\
\hline \multicolumn{5}{|c|}{ Zone avec toxicité ferreuse } \\
\hline \multirow{4}{*}{ DJ 684 D } & $15 \mathrm{JAR}$ & $100 \mathrm{a}$ & 93,9 a & 93,9 a \\
\hline & $30 \mathrm{JAR}$ & $100 \mathrm{a}$ & 90,9 a & 93,9 a \\
\hline & $60 \mathrm{JAR}$ & 98,9 a & 90,9 a & 69,2 b \\
\hline & $90 \mathrm{JAR}$ & 98,9 a & 90,9 a & $69,2 \mathrm{~b}$ \\
\hline \multirow{4}{*}{ Suakoko 8} & $15 \mathrm{JAR}$ & $100 \mathrm{a}$ & 98,9 a & 97,9 a \\
\hline & $30 \mathrm{JAR}$ & $100 \mathrm{a}$ & 95,9 a & 96,9 a \\
\hline & $60 \mathrm{JAR}$ & $100 \mathrm{a}$ & 95,9 a & 92,9 a \\
\hline & $90 \mathrm{JAR}$ & $100 \mathrm{a}$ & 95,9 a & 92,9 a \\
\hline \multirow{4}{*}{ Bouaké 189} & $15 \mathrm{JAR}$ & $100 \mathrm{a}$ & $100 \mathrm{a}$ & $100 a$ \\
\hline & $30 \mathrm{JAR}$ & $100 \mathrm{a}$ & $100 \mathrm{a}$ & $100 \mathrm{a}$ \\
\hline & $60 \mathrm{JAR}$ & $100 \mathrm{a}$ & $100 \mathrm{a}$ & $100 \mathrm{a}$ \\
\hline & $90 \mathrm{JAR}$ & $100 \mathrm{a}$ & $100 a$ & $100 a$ \\
\hline \multirow{4}{*}{ FAROX 521-356-H1 } & $15 \mathrm{JAR}$ & $100 a$ & $100 a$ & 93,9 a \\
\hline & $30 \mathrm{JAR}$ & 98,9 a & 98,9 a & 92,9 a \\
\hline & $60 \mathrm{JAR}$ & 98,9 a & $98,9 \mathbf{a}$ & 92,9 a \\
\hline & $90 \mathrm{JAR}$ & $98,9 a$ & $98,9 a$ & 92,9 a \\
\hline
\end{tabular}

*Les valeurs de la même ligne présentant les mêmes lettres ne sont pas statistiquement différentes au seuil LSD de $5 \%$ selon le test de Fisher.

Effet des amendements organiques sur le nombre moyen de talles, le diamètre moyen au collet, la hauteur moyenne et la biomasse végétale : Dans la zone sans toxicité ferreuse, pour le traitement témoin, il y'a eu une différence significative pour les variétés DJ $684 \mathrm{D}$ et Bouaké $189(\mathrm{p}<0.05)$. En comparant par variété, le $\mathrm{NmT}$ a significativement diminué avec l'amendement au biochar pour la variété Bouaké 189 dans la zone STox comme dans la zone Tox $(p<0.05)$. Pour l'amendement au compost et le témoin, il n'y a pas eu de différence significative entre les variétés $(p>$ 0.05 ) (Tableau 3). Pour ce qui est du diamètre moyen $(D m)$, aucune différence significative n'a été notée quel que soit l'amendement ou la variété dans la zone sans toxicité ferreuse comme dans la zone avec toxicité ferreuse $(P>0,05)$ (tableau 3$)$. Concernant la hauteur moyenne des plants, dans la zone sans toxicité ferreuse il n'y a pas de différence significative quel que soit l'amendement $(P>0,05)$ et quelle que soit la variété $(P>0,05)$. Cette même tendance a été obtenue dans la zone avec toxicité ferreuse entre les amendements $(P>0,05)$ et entre les variétés $(P>$ $0,05)$. Néanmoins, entre les deux zones il existe une différence significative de la hauteur moyenne des plants $(P<0,01)$. En prenant en compte l'amendement au biochar, nous constatons cependant que la variété Suakoko 8 a pratiquement la même hauteur dans les deux zones (tableau 3). Pour la biomasse sèche aérienne (BSA), il n'y a pas de différence significative ni entre les amendements $(P>0,05)$, ni au sein des variétés $(P>0,05)$ dans la zone sans toxicité ferreuse. Les mêmes résultats ont été obtenus dans la zone avec toxicité ferreuse entre les amendements $(P>0,05)$ et entre les variétés $(P>0,05)$. Ce qui nous permet de dire que les amendements organiques n'ont pas eu d'effet positif sur la production de biomasse aérienne (tableau 3). Par ailleurs, une différence significative de la biomasse sèche aérienne est constatée entre les deux zones $(P<0,01)$. 
Tableau 3: Valeurs moyennes de quelques paramètres de développement du riz cultivé en zone STox et Tox, amendé ou non au biochar ou au compost.

\begin{tabular}{|c|c|c|c|c|c|}
\hline \multirow{2}{*}{\multicolumn{2}{|c|}{ Traitements }} & \multicolumn{3}{|c|}{ Zone sans toxicité ferreuse } & \multirow[b]{2}{*}{ BSA } \\
\hline & & $\mathrm{NmT}$ & $\mathrm{Dm}$ & $\mathrm{Hm}$ & \\
\hline \multirow{4}{*}{ Témoin } & DJ 684 D & $9 \mathrm{bc}^{*}$ & $2,0 \mathrm{a}$ & $77,4 a b c$ & $7 a b c$ \\
\hline & Suakoko 8 & $11 a b$ & $2,5 \mathrm{a}$ & $78,0 \mathrm{abc}$ & $8,4 a b c$ \\
\hline & Bouaké189 & $14 a$ & $2,2 \mathrm{a}$ & $79,3 a b c$ & $7,5 \mathrm{abc}$ \\
\hline & FAROX 521-356-H1 & $10 a b$ & $1,8 \mathrm{a}$ & $75,2 \mathrm{abc}$ & $7,8 \mathrm{abc}$ \\
\hline \multirow{4}{*}{ Biochar } & DJ 684 D & $10 b$ & $2,3 a$ & $83,5 a b$ & $6,2 \mathrm{bc}$ \\
\hline & Suakoko 8 & $11 a b$ & $2,5 \mathrm{a}$ & $82,1 \mathrm{ab}$ & $7,5 \mathrm{abc}$ \\
\hline & Bouaké189 & $10 b$ & $2,4 \mathrm{a}$ & $85,0 \mathrm{a}$ & $8,2 a b c$ \\
\hline & FAROX 521-356-H1 & $11 \mathrm{ab}$ & $2,3 \mathrm{a}$ & $81,2 \mathrm{ab}$ & $8,8 \mathrm{abc}$ \\
\hline \multirow{4}{*}{ Compost } & DJ 684 D & $14 \mathrm{a}$ & $2,6 a$ & $85,4 a$ & $10,5 a b$ \\
\hline & Suakoko 8 & $13 a$ & $2,6 \mathrm{a}$ & $82,9 a b$ & $11,6 \mathrm{a}$ \\
\hline & Bouaké189 & $13 a$ & $2,7 \mathrm{a}$ & $81,2 a b$ & $10,2 a b c$ \\
\hline & FAROX 521-356-H1 & $13 a$ & $2,5 \mathrm{a}$ & $79,8 \mathrm{abc}$ & $8,7 a b c$ \\
\hline \multicolumn{6}{|c|}{ Zone avec toxicité ferreuse } \\
\hline \multirow{4}{*}{ Témoin } & DJ 684 D & $12 a b$ & $2,4 a$ & $75,8 \mathrm{abc}$ & $5,6 \mathrm{bcd}$ \\
\hline & Suakoko 8 & $9 \mathrm{bc}$ & $2,1 \mathrm{a}$ & 70,4 bc & $5,3 \mathrm{~cd}$ \\
\hline & Bouaké189 & $13 \mathrm{a}$ & $2,7 \mathrm{a}$ & $76,6 \mathrm{abc}$ & $5,6 \mathrm{bcd}$ \\
\hline & FAROX 521-356-H1 & $12 a b$ & $2,4 \mathrm{a}$ & $75,9 a b c$ & $4,7 \mathrm{~d}$ \\
\hline \multirow{4}{*}{ Biochar } & DJ 684 D & $10 \mathrm{~b}$ & $2,1 \mathrm{a}$ & $66,9 \mathrm{c}$ & $5,6 \mathrm{bcd}$ \\
\hline & Suakoko 8 & $9 \mathrm{bc}$ & $2,2 \mathrm{a}$ & $81,5 a b$ & $7,3 \mathrm{abc}$ \\
\hline & Bouaké189 & $9 \mathrm{bc}$ & $2,0 \mathrm{a}$ & 70,8 bc & $5,4 \mathrm{bcd}$ \\
\hline & FAROX 521-356-H1 & $10 \mathrm{~b}$ & $2,4 a$ & $68,7 \mathrm{c}$ & $8,2 \mathrm{abc}$ \\
\hline \multirow{4}{*}{ Compost } & DJ 684 D & $9 \mathrm{bc}$ & $2,1 \mathrm{a}$ & $70,9 \mathrm{bc}$ & $4,7 \mathrm{~d}$ \\
\hline & Suakoko 8 & $7 c$ & $2,2 \mathrm{a}$ & $58,7 d$ & $6,3 \mathrm{bc}$ \\
\hline & Bouaké189 & $9 \mathrm{bc}$ & $1,8 \mathrm{a}$ & $72,2 \mathrm{abc}$ & $5,9 \mathrm{bcd}$ \\
\hline & FAROX 521-356-H1 & $11 \mathrm{ab}$ & $2,0 \mathrm{a}$ & $68,1 \mathrm{c}$ & 6,4 bc \\
\hline
\end{tabular}

*Les valeurs de la même colonne affectées d'une même lettre ne sont pas statistiquement différents au seuil LSD de $5 \%$ selon le test de Fisher.

Effet des amendements organiques sur le poids de mille grains, le poids moyen des panicules et le rendement en grains: II n'y a pas de différence significative pour le poids de mille grains (PMG) entre les amendements $(P>0,05)$ et entre les variétés $(P>$ $0,05)$ dans la zone STox. Dans la zone Tox, la même tendance a été observée entre les amendements $(P>$ $0,05)$. Cependant, il y'a une différence significative entre les variétés $(P<0,01)$ et entre les deux zones $(P$ $<0.01)$. La toxicité ferreuse a eu un effet négatif sur le poids de mille grains (Tableau 4). L'analyse de la variation du poids moyen des panicules (PmP) ne montre pas de différence significative dans la zone
STOX pour tous les traitements ( $P>0,05$, Tableau 4). Cette même tendance a été obtenue dans la zone Tox au niveau des amendements $(P>0,05)$. Par ailleurs, les variétés Bouake 189 et FAROX 521-356-H1 ont eu PmP statistiquement supérieur à celui de DJ 684D au niveau du témoin non-amendé dans la zone Tox. De même, dans la zone STox la variété Suakoko 8 a eu un PmP statistiquement supérieur à celui de DJ $684 \mathrm{D}$ (Tableau 4). Aucune différence significative du rendement en grains (Rdt) n'a été notée entre les deux zones $(P>0,05)$ quel que soit l'amendement apporté ou la variété cultivée (Tableau 4). 
Tableau 4: Poids de mille grains, poids moyen des panicules et rendement en grains de quatre variétés de riz cultivées en zone STox et Tox, amendées ou non au biochar ou au compost.

\begin{tabular}{|c|c|c|c|c|}
\hline \multicolumn{5}{|c|}{ Zone sans toxicité ferreuse } \\
\hline Traitements & & PMG & PmP & Rdt \\
\hline \multirow{4}{*}{ Témoin } & DJ 684 D & $18,6 \mathrm{bcd}^{*}$ & $62,3 b c$ & $1,8 \mathrm{a}$ \\
\hline & Suakoko 8 & $19 \mathrm{bcd}$ & $77,6 \mathrm{abc}$ & $2,6 \mathrm{a}$ \\
\hline & Bouaké 189 & 17,3 de & $69 a b c$ & $1,4 \mathrm{a}$ \\
\hline & FAROX 521-356-H1 & 19 bcd & $75 \mathrm{abc}$ & $1,9 a$ \\
\hline \multirow{4}{*}{ Biochar } & DJ 684 D & $19,6 \mathrm{abc}$ & $75,6 \mathrm{abc}$ & $2,1 \mathrm{a}$ \\
\hline & Suakoko 8 & $19 \mathrm{bcd}$ & $87,6 a b c$ & $2,3 \mathrm{a}$ \\
\hline & Bouaké 189 & $19 \mathrm{bcd}$ & $87 a b c$ & $1,9 \mathrm{a}$ \\
\hline & FAROX 521-356-H1 & $19 \mathrm{bcd}$ & $101,6 \mathrm{ab}$ & $2,4 \mathrm{a}$ \\
\hline \multirow{4}{*}{ Compost } & DJ 684 D & $17 \mathrm{e}$ & $97 \mathrm{ab}$ & $2,7 \mathrm{a}$ \\
\hline & Suakoko 8 & $19 \mathrm{bcd}$ & $113,3 a$ & $3,4 \mathrm{a}$ \\
\hline & Bouaké 189 & $19 \mathrm{bcd}$ & $95 \mathrm{ab}$ & $3,0 \mathrm{a}$ \\
\hline & FAROX 521-356-H1 & $18 \mathrm{bcd}$ & $95,7 \mathrm{ab}$ & $1,9 a$ \\
\hline & \multicolumn{4}{|c|}{ Zone avec toxicité ferreuse } \\
\hline \multirow{4}{*}{ Témoin } & DJ 684 D & 18,3 cde & $43 c$ & $2,4 \mathrm{a}$ \\
\hline & Suakoko 8 & $21,6 a b$ & $68 \mathrm{abc}$ & $1,9 \mathrm{a}$ \\
\hline & Bouaké 189 & $18,6 \mathrm{bcd}$ & $105,6 a b$ & $3,0 \mathrm{a}$ \\
\hline & FAROX 521-356-H1 & $18,6 \mathrm{bcd}$ & 58 bc & $2,5 \mathrm{a}$ \\
\hline \multirow{4}{*}{ Biochar } & DJ 684 D & $20,3 a b c$ & $82,3 \mathrm{abc}$ & $2,2 \mathrm{a}$ \\
\hline & Suakoko 8 & $22,6 \mathrm{a}$ & $83,6 a b c$ & $2,2 \mathrm{a}$ \\
\hline & Bouaké 189 & $20,3 a b c$ & $66 \mathrm{abc}$ & $2,0 \mathrm{a}$ \\
\hline & FAROX 521-356-H1 & $21,6 \mathrm{ab}$ & $101,3 \mathrm{ab}$ & $2,6 \mathrm{a}$ \\
\hline \multirow{4}{*}{ Compost } & DJ 684 D & $20 a b c$ & $80 a b c$ & $1,6 \mathrm{a}$ \\
\hline & Suakoko 8 & $21,6 a b$ & $83 a b c$ & $2,0 \mathrm{a}$ \\
\hline & Bouaké 189 & $19,0 \mathrm{bcd}$ & 59,6 bc & $1,7 \mathrm{a}$ \\
\hline & FAROX 521-356-H1 & $21,3 a b c$ & $80,3 a b c$ & $3,0 \mathrm{a}$ \\
\hline
\end{tabular}

* Les valeurs de la même colonne affectées d'une même lettre ne sont pas statistiquement différents au seuil LSD de 5\% selon le test de Fisher.

\section{DISCUSSION}

Le $\mathrm{pH}$ va-t-il été modifié suite à l'apport d'amendement compost ou biochar?: Une augmentation du pH précisément à 15 JAR dans les deux zones conduisant à un caractère modérément acide des sols a été notée. Toutefois, la diminution significative notée à $90 \mathrm{JAR}$ en zone STox avec le compost au niveau des horizons $0-10 \mathrm{~cm}$ et $10-30 \mathrm{~cm}$, peut être liée à l'effet tampon de la matière organique qui a été beaucoup plus intense en surface dans les premières semaines. Un $\mathrm{pH}$ voisin de la neutralité constitue un atout pour une meilleure absorption racinaire des éléments nutritifs (Ognalaga et al., 2015). $\mathrm{Au}$ fil des temps, avec l'engorgement en eau, la décomposition de cette matière organique a acidifié beaucoup plus le milieu et a fait baisser le $\mathrm{pH}$. L'acidité des sols a été associée à l'apparition de la toxicité ferreuse en fin de saison des pluies (AfricaRice, 2006). Ouédraogo (2010) a noté une augmentation du pH quelques jours après amendement suivie d'une diminution progressive en fin de culture. Par ailleurs, avec la saison pluvieuse, l'inondation de rizières, provoque une augmentation rapide et importante du fer ferreux élément toxique pour le riz rabaissant le $\mathrm{pH}$ et entrainant la libération du fer (Marius et Cheval, 1980, AfricaRice, 2006). Ces résultats confirment le travail de Prade et al., (1990) qui soutient que le taux d'acidité 
des bas-fonds dans la Région Naturelle de la Casamance particulièrement dans les parties Basse et Moyenne, est fonction de la saison.

Les amendements organiques ont-ils amélioré les paramètres agro-morphologiques et le rendement du riz en zone avec toxicité ferreuse ?: Pour trois des quatre variétés (Bouaké 189, Suakoko 8 et FAROX 521-356-H1), le taux de survie des plants n'est pas statistiquement différent en comparant les deux zones. La présence de fer n'a pas impacté négativement le taux de survie des plants. Ceci peut être dû à la tolérance $(<300 \mathrm{ppm})$ des variétés utilisées face à cette contrainte et à la lame d'eau qui dilue la concentration des éléments solubles. Diouf (1988) montre que le $\mathrm{pH}$ des sols sulfatés acides, généralement bas, ne constitue pas un facteur limitant à la survie et/ou à la croissance des plants du riz, car, avec les apports d'eaux, ce pH in situ, se rétablit assez rapidement, à des valeurs comprises entre 5,5 et 7,0. Toutefois, pour la variété DJ $684 \mathrm{D}$, la présence de fer réduit la survie et le développement des plantes. Ces résultats sont en accord avec ceux de Onaga et al., 2012). L'amélioration de la hauteur de plants de la variété Suakoko 8 dans la zone avec toxicité ferreuse avec l'apport du biochar pourrait être due au fait que ces variétés ont pu bénéficier des éléments nutritifs avec les amendements organiques (Badji, 2011). Ces amendements leur ont permis de booster leur croissance; ce qui aurait permis d'avoir la même performance aussi bien dans la zone avec toxicité ferreuse que dans la zone sans toxicité ferreuse. L'ajout de biochar aux substrats favorise une meilleure croissance des plants comme l'a souligné Butnan et al. (2015). Les résultats Biedermann \& Harpole, (2013) ont aussi confirmé que l'amendement en biochar peut avoir un effet bénéfique sur la croissance des plantes.

Les variétés utilisées sont résistantes à la toxicité ferreuse. Ce faisant, leur optimum de résistance $(<300$ ppm) pourrait se situer à une certaine concentration de

\section{CONCLUSIONS ET PERSPECTIVES}

L'apport du compost a conduit à une diminution $\mathrm{du} p \mathrm{pH}$ à $90 \mathrm{JAR}$ dans les horizons $0-10 \mathrm{~cm}$ et $10-30 \mathrm{~cm}$ en zone sans toxicité ferreuse. Cependant, dans cette même zone, l'amendement au compost a permis une nette augmentation du nombre de talles pour tous les variétés. La hauteur des plantes a été influencée positivement avec l'apport du biochar dans la zone avec toxicité ferreuse pour la variété Suakoko 8. La toxicité ferreuse a impacté négativement le taux de fer. De ce fait, ces variétés ne pourront pas se développer de manière optimale dans un milieu sans toxicité ferreuse. Ce qui pourrait expliquer l'obtention des valeurs similaires en zone sans toxicité ferreuse tout comme en zone avec toxicité ferreuse. Le constat fait sur le taux de survie des plants et la biomasse végétale des variétés Suakoko 8, DJ 6684 D et FAROX 521-356-H1 des sols amendées au biochar ou au compost atteste que ces dernières ont été affectées par la toxicité ferreuse. Ce résultat confirme celui de ADRAO, (2006) qui a trouvé qu'une absorption excessive d'ions ferreux $\left(\mathrm{Fe}^{2+}\right)$ par les racines et leur translocation acropète par le flux de xylème vers les organes aériens porte atteinte au bon développement des plants de riz. Abu et al. (1989) rapportent que la toxicité ferreuse pendant les stades végétatifs entraine une réduction de la matière végétale de riz. De plus, dans le cas d'une toxicité se produisant pendant le stade plantule, la formation de talles et la proportion de talles productives peuvent être sévèrement réduites (Cheema et al., 1990). Le poids des panicules pourrait être affecté par la toxicité ferreuse en fonction du seuil de tolérance de la variété et de la date de manifestation de cette contrainte. Des études ont montré que la toxicité ferreuse se produisant en fin de phase végétative ou en début de phase reproductive, fait chuter le nombre de panicule (Singh et al., 1992). Virmani, (1977) soutient que la stérilité des épilés augmente et les étapes de floraison et de maturation peuvent être retardés d'environ 20-25 jours, ceci en condition de toxicité ferreuse. Pour ce qui est du poids de 1000 grains, nous constatons que quelle que soit la zone, l'amendement apporté ou la variété cultivée, ce paramètre n'est pas différent significativement entre les deux zones. Ce qui pourrait être attribué aux caractéristiques variétales. Andrianjatovo, (2012) soutient que le poids de 1000 grain est un caractère variétal qui est difficilement influencé par les pratiques culturales.

survie des variétés comme DJ $684 \mathrm{D}$, Suakoko 8 et FAROX 521-356- $\mathrm{H} 1$ et la production de biomasse des variétés DJ 684 D et Suakoko 8. II serait intéressant d'évaluer les effets à long terme des amendements organiques sur le rendement du riz mais aussi sur la modification des propriétés physicochimiques du sol influençant la disponibilité en nutriments du sol. 
Remerciements : Nous tenons à remercier Landing Badji pour son rôle important dans la réalisation de ce travail.

\section{REFFERENCES}

Abu MB, Tucker ES, Harding SS, Sesay JS, 1989. Cultural practices to reduce iron toxicity in rice. International Rice Research Newsletter 14 (1), $19 p$.

ADRAO, 2001. Resultats definitifs du rapport annuel de la campagne agricole 2001/2002. Association pour le développement de la riziculture en Afrique de l'Ouest. http://www.africarice.org

ADRAO, 2006. Toxicité ferreuse dans les systèmes à base riz d'Afrique de l'ouest, Centre du riz pour l'Afrique (ADRAO) Cotonou, Bénin. 196 pp. http://www. africarice.org

AfricaRice, 2006. Iron Toxicity in Rice-based Systems in West Africa. Africa Rice Center (WARDA). Cotonou, Benin, 175pp.

APRAO, 2012. Projet Amélioration de la Production du Riz en Afrique de l'Ouest en réponse à la Flambée des Prix des Denrées Alimentaires (GCP/RAF/453/SPA) / Composante Sénégal, Rapport Provisoire 2012, 30p. http://www.fao.org

Andrianjatovo, 2012. Effet de la densité des semences de riz sur le développement des plants et sur le rendement: Cas des hautes terres, Mémoire de fin d'études en vue d'obtenir le diplôme d'ingénieur Agronome, Université D'Antananarivo, $74 p$.

Badji A, 2011. Effets du biochar sur les activités microbiologiques du sol sous forts intrants azotes (maraichage).http://horizon.docum entation.ird.fr/exl-doc/pleins_textes/divers1306/010058144.pdf

Biederman LA and Harpole WS, 2013. Biochar and its effects on plant productivity and nutrient cycling: a meta-analysis. GCB Bioenergy 5, 202-214. doi:10.1111/gcbb.12037

Butnan S, Deenik JL, Toomsan B, Antal MJ, Vityakon $P$, 2015. Biochar characteristics and application rates affecting corn growth and properties of soils contrasting in texture and mineralogy. Geoderma. 237: 105-116.

Cheng $\mathrm{CH}$, Lehmann J, Engelhard MH, 2008. Natural oxidation of black carbon in soils: Changes in molecular form and surface charge along a climosequence. Geochimica and Cosmochimica Acta 72: 1598-1610. doi:10.1016/j.gca.2008.01.010
Criscuoli I, 2016. Stabilité du charbon végétal (biochar) dans le sol et impact sur la productivité et les cycles des nutriments des prairies alpines. Géochimie. Université Pierre et Marie Curie Paris VI, 2016. Français. 20-22 p. https://tel.archives-ouvertes.fr

Dai Z, Zhang X, Tang C, Muhammad N, Wu J, Brookes PC, Xu J, 2017. Potential role of biochar in decreasing soil acidification - A critical review. Science of the Total Environment 581: 601611.

Del Villar PM, Bauer JM, Maiga A, Ibrahim L, 2011.Crise rizicole, évolution des marchés et sécurité alimentaire en Afrique de l'Ouest. Ministère des Affaires étrangères. France. 61 p. https://documents.wfp.org

Ding Y, Liu Y, Liu S, Li Z, Tan X, Huang X, Zeng G, Zhou $L$, Zheng $B, 2016$. Biochar to improve soil fertility. A review. Agronomy for Sustainable Development 36: 36.

Kelly V, Reardon T, Fall A, Diagana BN, 1993. Impact des politiques de prix et de revenus sur la consumation et l'offre des produits agricoles. Volume $1 \& 2$, partie I \& II. Rapport final du Projet ISRA/IFPRI. $93 \mathrm{p}$.

Kite R, 1993. Senegal: Implications for the Rice Policy Dialogue. USAID/Dakar. $43 p$

Kolton M, Graber ER, Tsehansky L, Elad Y, Cytryn E, 2017. Biochar-stimulated plant performance is strongly linked to microbial diversity and metabolic potential in the rhizosphere. New Phytologist 213 : 1393-1404.

Lacharme M, 2001. "Fascicule 2 ». Le plant de riz, données morphologiques et cycle de la plante. Mémento Technique de Riziculture, 22p. Site: www.aridafrique.org.

Laird DA, 2008. The Charcoal Vision: A Win-Win-Win Scenario for Simultaneously Producing Bioenergy, Permanently Sequestering Carbon, while Improving Soil and Water Quality. Agronomy Journal 100: 178-181. doi:10.2134/agronj2007.0161

Laurin-Lanctôt S, 2015. Effet de l'amendement en biochar des sols biologiques pour une culture de tomates sous serre : rétention en nutriments, activité biologique et régie de fertilisation. $146 \mathrm{p}$. 
Onaga G, Edema R, Asea G, 2012. Tolerance of rice germplasm to iron toxicity stress and the relationship between tolerance, $\mathrm{Fe} 2+, \mathrm{P}$ and $\mathrm{K}$ content in the leaves and roots. Archives of Agronomy and Soil Science, 59:1-17. http://doi.org/10.1080/03650340.2011.622751

Ognalaga M, Djegui PIO, Lekambou JM, Poligui RN, 2015. Effet des écumes à cannes à sucre, de la poudre et du compost de à base de Chromolaena odorata (L.) King R.M. \& H.E. Rob sur la croissance de l'oseille de Guinée (Hibiscus sabdariffa L.). Int. J. Biol. Chem. Sci. 9: 2507-2519, https://www.ajol.info , index. php

PLD, 2010. Plan local de développement communauté rurale de Mangagoulack réalisé par le groupe OCC avec l'appui financier du PNDL, 97p

PNAR, 2009. Stratégie Nationale de Développement de la Riziculture. Programme National d'autosuffisance en riz. Ministère de l'Agriculture. Dakar, Sénégal. 26 p. https://www.ipar.sn

Prade K, Ottow JCG, Jacq VA, Malouf GLJY, 1990. Relations entre les propriétés des sols de rizières inondées et la toxicité ferreuse en basse Casamance (Sénégal). ORSTOM, XXV, 453-474p. https://core.ac.uk

Sagna P, 2005. Dynamique du climat et son évolution récente dans la partie ouest de l'Afrique occidentale, Thèse de doctorat d'Etat ES Lettre, UCAD, Faculté des lettre et Sciences Humaine, Département de Géographie, 790p.

Seck M, 2015. Autosuffisance en riz : 600000 t de riz blanc attendues au Sénégal selon Macky Sall. In Agri infos Mensuel d'informations Agricoles et rurales - 9e Année - $\mathrm{N}^{\circ} 81$ - Issn 08508844. Avril 2015. 4 p. www.inter-reseaux.org

Singh BP, Das M, Prasad RN, Ram M, 1992. Characteristics of Fe-toxic soils and affected plants and their correction in acid Haplaquents of Meghalaya. Int. Rice Res. Newsl. 17: 18-19.

Virmani SS, 1977. Varietal tolerance of rice to iron toxicity in Liberia. Int. Rice Res. Newsl. 2: 4-5.

Yao Y, Gao B, Zhang M, Inyang M, Zimmerman AR, 2012. Effect of biochar amendment on sorption and leaching of nitrate, ammonium, and phosphate in a sandy soil. Chemosphere. 89: 1467-1471. 